\title{
A DISCRETE ANALOGUE OF THE HARMONIC MORPHISM AND GREEN KERNEL COMPARISON THEOREMS
}

\author{
HAJIME URAKAWA \\ Mathematics Laboratories, Graduate School of Information Sciences, Tohoku University, Katahira, \\ 2-1-1, Sendai, 980-8577, Japan \\ e-mail:urakawa@math.is.tokoku.ac.jp
}

(Received 24 August, 1998; revised 26 May, 1999)

\begin{abstract}
We give a discrete analogue of the harmonic morphism between two Riemannian manifolds. Roughly speaking, this is a mapping between two graphs preserving local harmonic functions. We characterize harmonic morphisms in terms of horizontal conformality. Many examples including coverings, non-complete extended p-sums and collapsings are given. Introducing the horizontal and vertical Laplacians, the Green kernel estimates are obtained for the harmonic morphism. As applications, a general and sharp estimate of the Green kernel for an infinite tree is obtained.
\end{abstract}

1991 Mathematics Subject Classification. 05 C50, 58 E20.

1. Introduction. The last decade has seen an increasing interest in Discrete Potential Theory; that is, the study of Laplacians, harmonic functions, Green's functions, etc, associated with transition operators on graphs. The basic features remain the same as in the continuous set-up, but one now has the advantage that they are not obscured by technicalities stemming from local geometry and computations on manifolds. It is therefore legitimate to take objects from continuous Potential Theory and carry them over to the discrete setting to shed new light on the original issue.

In this paper, we want to discretize concepts of harmonic morphisms between Riemannian manifolds; i.e., mappings that preserve harmonicity locally to graphs. For Riemannian manifolds, harmonic morphisms were introduced by Fugulede [3] and Ishihara [4] in the late 1970's.

A graph $G=(V, E)$ is a collection of the set $V$ of vertices and the set $E$ of edges connecting two vertices. For two graphs, $G_{1}=\left(V_{1}, E_{1}\right)$ and $G_{2}=\left(V_{2}, E_{2}\right)$, a mapping $\varphi: V_{1} \rightarrow V_{2}$ is called a harmonic morphism if $f \circ \varphi$ is harmonic at $x \in V_{1}$ whenever $f$ is harmonic at $\varphi(x) \in V_{2}$.

The stochastic meaning of harmonic morphism is that it is a mapping sending the Brownian motion on a Riemannian manifold into another such under a suitable random time change, and our discrete harmonic morphism is a mapping sending the random walk on a graph into that of another graph.

In Section 2, we characterize harmonic morphisms in terms of horizontal conformality, (cf. Theorem 2.5) which enables us to give several examples of harmonic morphisms. As an application, we obtain the Green kernel comparison theorem (cf. Theorem 3.3) which states that, for a harmonic morphism $\varphi: G_{1} \rightarrow G_{2}$ between two infinite graphs $G_{i}(i=1,2)$,

$$
g_{1}(x, y) \leq \frac{m(y)}{m_{h}(y)} g_{2}(\varphi(x), \varphi(y)),
$$


for all $x$ and $y$ in $V_{1}$. Here $g_{i}$ are Green's functions on $G_{i}$. This Green kernel comparison theorem enables us to estimate Green's functions of several infinite graphs explicitly in Section 4.

2. Harmonic Morphisms. In this section, we define two different notions, a harmonic morphism and a horizontally conformal map between two graphs. We shall prove the basic facts about harmonic morphisms and state the main theorem (cf. Theorem 2.5).

It is well known (cf. [2], [7], [10], [11]) that the discrete Laplacian for a graph $G=(V, E)$ acting on the space $C(V)$ of real valued functions on $V$ can be defined.

\section{DEFINITION 2.1}

$$
\Delta f(x)=f(x)-\frac{1}{m(x)} \sum_{z \sim x} f(z), \quad f \in C(V),
$$

where $z \sim x$ means that $z$ is incident to $x$.

Let us give an orientation on each edge of $G=(V, E)$, once and for all. For $e \in E$, let $o(e), t(e)$ be the origin and terminal of $e$, respectively. For $e \in E$, let $\bar{e}$ be the reverse of $e$; that is, the origin of $\bar{e}$ is $t(e)$ and the terminal of $\bar{e}$ is $o(e)$. Let $\mathbf{E}$ be the set of oriented edges of $G$. Then $\mathbf{E}=\{e, \bar{e} ; e \in E\}$. A 1-form of a graph $G=(V, E)$ is a function $\omega$ on $\mathbf{E}$ satisfying that

$$
\omega(e)=-\omega(\bar{e}), \quad \forall e \in \mathbf{E} .
$$

We denote by $A^{1}(G)$ the space of all 1-forms on $G$. Also let $A^{0}(G)=C(V)$.

The differential $d: A^{0}(G) \rightarrow A^{1}(G)$ by

$$
d f(e)=f(t(e))-f(o(e)), \quad f \in A^{0}(G),
$$

and the inner products on $A^{0}(G)$ and $A^{1}(G)$ are defined by

$$
\begin{aligned}
\left(f_{1}, f_{2}\right) & =\sum_{x \in V} f_{1}(x) f_{2}(x), \quad f_{1}, f_{2} \in A^{0}(G), \\
\left(\varphi_{1}, \varphi_{2}\right) & =\sum_{e \in E} \varphi_{1}(e) \varphi_{2}(e), \quad \varphi_{1}, \varphi_{2} \in A^{1}(G) .
\end{aligned}
$$

The co-differential $\delta: A^{1}(G) \rightarrow A^{0}(G)$ is defined by

$$
m(x) \delta(\omega)(x)=-\sum_{e \in \mathbf{E}, o(e)=x} \omega(e)=\sum_{e \in E, t(e)=x} \omega(e)-\sum_{e \in E, o(e)=x} \omega(e), \quad x \in V,
$$

for all $\omega \in A^{1}(G)$. It is known that for all $f \in A^{0}(G)$ and $\omega \in A^{1}(G)$

$$
\delta(d f)(x)=\Delta f(x) \quad \text { and } \quad(d f, \omega)=(f, \delta \omega) .
$$


A function $f \in C(V)$ is said to be harmonic at $x$ if

$$
f(x)=\frac{1}{m(x)} \sum_{z \sim x} f(z),
$$

which is equivalent to $\Delta f(x)=0$. The spaces of harmonic functions and harmonic 1 -forms are given as

$$
\mathbf{H}^{0}(G)=\left\{f \in A^{0}(G) ; \Delta f=0\right\}, \quad \mathbf{H}^{1}(G)=\left\{\omega \in A^{1}(G) ; \delta \omega=0\right\},
$$

Also the $q$-th Betti numbers of a graph $G=(V, E)$ are defined by $b_{q}(G)=\operatorname{dim} \mathbf{H}^{q}(G), \quad(q=0,1)$, respectively.

Let us now define a harmonic morphism and a horizontally conformal map. Let $G_{i}=\left(V_{i}, E_{i}\right), i=1,2$ be two graphs and $\varphi: V_{1} \rightarrow V_{2}$ a mapping.

Definition 2.2. (1) $\varphi$ is said to be a harmonic morphism of $G_{1}$ to $G_{2}$ if for any $y \in V_{2}$ belonging to the image of $\varphi$, say $y=\varphi(x), x \in V_{1}$, any function $f$ on $V_{2}$ that is harmonic at $y$, the composition $f \circ \varphi$ is harmonic at $x$.

(2) $\varphi$ is said to be horizontally conformal if the following two conditions hold.

(a) For all $z, x \in V_{1}, \varphi(z) \sim \varphi(x)$ or $\varphi(z)=\varphi(x)$ whenever $z \sim x$.

(b) For all $y \in V_{2}$ and $x \in \varphi^{-1}(y), \#\left\{z \in \varphi^{-1}\left(y^{\prime}\right) ; z \sim x\right\}$ is constant in $y^{\prime} \in V_{2}$ with $y^{\prime} \sim y$.

The conditions of horizontal conformality correspond to the surjectivity of the differential on the non-vanishing set of the energy density, and the conformality on the horizontal spaces. The following Figure 1 illustrates harmonic morphism and horizontal conformality.

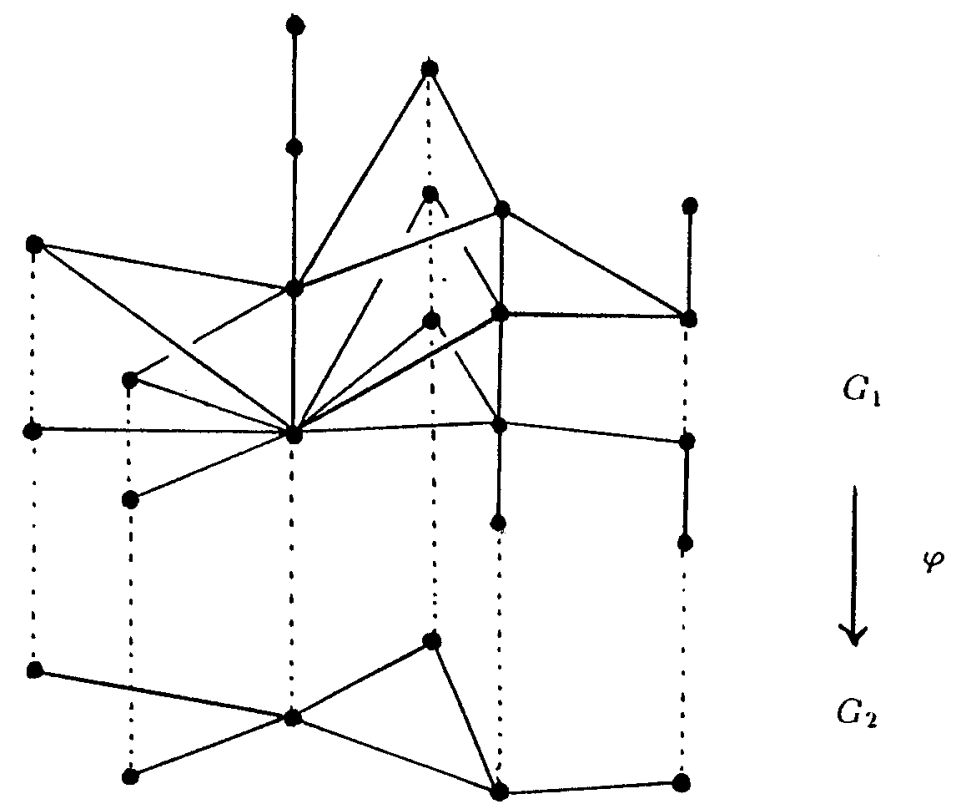

Figure 1 
REMARK 2.3. The probabilistic meaning of a harmonic morphism is the following. Let $Z_{i}$ be the random walks, in general, the Markov process corresponding to graphs $G_{i}=\left(V_{i}, E_{i}\right), i=1,2$. Let $\varphi: V_{1} \rightarrow V_{2}$ be a mapping. Then $\varphi$ is a harmonic morphism of $G_{1}$ to $G_{2}$ if and only if $Z_{1}$ projects to $Z_{2}$ by $\varphi$ under a suitable random time change on the set of non-negative integers.

Definition 2.4. Let $\varphi: V_{1} \rightarrow V_{2}$ be a harmonic morphism of $G_{1}$ to $G_{2}$. For $x \in V_{1}$, let us define the vertical degree $m_{v}(x)$ and the horizontal degree $m_{h}(x)$ by

$$
\begin{aligned}
& m_{v}(x)=\#\left\{z \in V_{1} ; z \in \varphi^{-1}(\varphi(x)) \text { and } z \sim x\right\}, \\
& m_{h}(x)=\#\left\{z \in V_{1} ; z \notin \varphi^{-1}(\varphi(x)) \text { and } z \sim x\right\} .
\end{aligned}
$$

Clearly we have

$$
m(x)=m_{v}(x)+m_{h}(x) .
$$

Our main result can be stated as follows.

Theorem 2.5. Let $G_{i}=\left(V_{i}, E_{i}\right), i=1,2$ be two graphs and $\varphi: V_{1} \rightarrow V_{2}$ an onto mapping. Then $\varphi$ is a harmonic morphism if and only if $\varphi$ is horizontally conformal.

To prove Theorem 2.5, for two graphs $G_{i}=\left(V_{i}, E_{i}\right), i=1,2$ let $d_{i}(x, y)$ be the graph distance of $G_{i}$ and $m_{i}(x)$ the degree of $x \in V_{i}$ for $G_{i}$. We also denote by $N_{i}(x)$, the set of neighbors of $x$ and $x$ itself, where $x \in V_{i}$.

Lemma 2.6. Let $\varphi: V_{1} \rightarrow V_{2}$ be a harmonic morphism of $G_{1}$ to $G_{2}$. Then, for all $x \in V_{1}$ and $y \in N(x), d_{2}(\varphi(x), \varphi(y)) \leq 1$. Therefore $\varphi$ is distance decreasing i.e.,

$$
d_{2}(\varphi(x), \varphi(y)) \leq d_{1}(x, y),
$$

for all $x, y \in V_{1}$.

Proof. Let $v=\varphi(x)$. Consider a function $f$ on $V_{2}$ defined by

$$
f(w)= \begin{cases}1 & w \in N_{2}(v) \\ 0 & \text { otherwise. }\end{cases}
$$

Then, by definition, $f$ is harmonic at $v$. Since $\varphi$ is a harmonic morphism, $f \circ \varphi$ is harmonic at $x$. Therefore, we have

$$
1=f \circ \varphi(x)=\frac{1}{m_{1}(x)} \sum_{y \sim x} f \circ \varphi(y)=\frac{1}{m_{1}(x)} \#\left\{y \in V_{1} ; y \sim x, \varphi(y) \in N_{2}(v)\right\},
$$

which implies that $m_{1}(x)=\#\left\{y \in V_{1} ; y \sim x, \varphi(y) \in N(v)\right\}$. It follows that $\varphi(y) \in N_{2}(v)$ for all $y \in V_{1}$ with $y \sim x$.

To see the last statement, assume $d\left(x, x^{\prime}\right)=n$, for $x, x^{\prime} \in V_{1}$ and take a shortest path $c=\left[z_{1}, z_{2} \cdots, z_{n}\right] \quad\left(z_{1}=x\right.$ and $\left.z_{n}=x^{\prime}\right)$ connecting $x$ and $x^{\prime}$ with length $L(c)=n$. Then $\varphi(c)=\left[\varphi\left(z_{1}\right), \varphi\left(z_{2}\right), \cdots, \varphi\left(z_{n}\right)\right]$ is a path in $G_{2}$ connecting $\varphi(x)$ and $\varphi(y)$, by virtue of the first statement. Therefore, we have 


$$
d\left(x, x^{\prime}\right)=L(c) \geq L(\varphi(c))=d\left(\varphi(x), \varphi\left(x^{\prime}\right)\right) .
$$

Lemma 2.7. Let $\varphi: V_{1} \rightarrow V_{2}$ be a harmonic morphism of $G_{1}$ to $G_{2}$. Let $v=\varphi(x), x \in V_{1}$ and $w \in V_{2}$ with $w \in N(v)$. Then,

$$
\#\left\{y \in V_{1} ; y \sim x, \varphi(y)=w\right\}= \begin{cases}m_{v}(x), & \text { if } w=v, \\ \frac{m_{h}(x)}{m_{2}(v)}, & \text { if } w \sim v,\end{cases}
$$

which depends only on $x$ if $w \sim v$.

Proof. For $w \in N(v)$, we denote by

$$
k_{w}(x)=\#\left\{y \in V_{1} ; y \sim x, \varphi(y)=w\right\} .
$$

Then by definition we have $k_{v}(x)=m_{v}(x)$. If $w \sim v$, define a function $f_{w}$ on $V_{2}$ by

$$
\left\{\begin{array}{l}
f_{w}(w)=1, \\
f_{w}(v)=\frac{1}{m_{2}(v)} \\
f_{w}=0 \text { otherwise. }
\end{array}\right.
$$

Since $f_{w}$ is harmonic at $v, f_{w} \circ \varphi$ is harmonic at $x$, by definition. Therefore, we have

$$
\begin{aligned}
\frac{m_{1}(x)}{m_{2}(v)} & =m_{1}(x) f_{w}(v)=m_{1}(x) f_{w} \circ \varphi(x) \\
& =\sum_{y \sim x} f_{w} \circ \varphi(y) \\
& =\sum_{y \sim x, \varphi(y)=v} f_{w} \circ \varphi(y)+\sum_{y \sim x, \varphi(y)=w} f_{w} \circ \varphi(y) \\
& =\frac{m_{v}(x)}{m_{2}(v)}+k_{w}(x),
\end{aligned}
$$

which implies the desired result.

By Lemmas 2.6 and 2.7, we have immediately the following result.

Proposition 2.8. Let $\varphi: V_{1} \rightarrow V_{2}$ be a harmonic morphism of $G_{1}$ to $G_{2}$ and $y=\varphi(x) \in V_{2}$ with $x \in V_{1}$. Then we have $\varphi(N(x))=\{\varphi(x)\}$ or $N(\varphi(x))$, and

$$
m(x) \geq m_{h}(x) \geq m(y) .
$$

Remark 2.10. The meaning of (2.9) in Proposition 2.8 is the following. If we define the curvature at $x \in V$ of a graph $G=(V, E)$ by $K_{G}(x)=2-m(x)$, then

$$
K_{G_{1}}(x) \leq K_{G_{2}}(\varphi(x)), \text { for all } x \in V_{1} .
$$


It is known that the analogy holds for a harmonic morphism between two Riemannian manifolds.

Lemma 2.11. Assume that $\varphi$ is horizontally conformal; i.e., (a) for all $x \in V_{1}$ and $y \in V_{1}$ with $y \sim x, \varphi(y) \in N(\varphi(x))$; (b) for all $x \in V_{1}$ and $w \in V_{2}$ with $w \sim \varphi(x)$, $k_{w}(x)=\#\left\{y \in V_{1} ; y \sim x, \varphi(y)=w\right\}$ depends only on $x$. Then $\varphi$ is a harmonic morphism.

Proof. For any $x \in V_{1}, k_{w}(x)=\#\left\{y \in V_{1} ; y \sim x, \varphi(y)=w\right\}$ depends only on $x$, and so we denote $k(x)=k_{w}(x)$. Put $v=\varphi(x)$. By (a), we have

$$
\begin{aligned}
m_{1}(x) & =m_{v}(x)+\sum_{w \in V_{2}, w \sim v} \#\left\{y \in V_{1} ; y \sim x, \varphi(x)=w\right\} \\
& =m_{v}(x)+m_{2}(v) k(x) .
\end{aligned}
$$

Now assume that $\varphi$ is harmonic at $v=\varphi(x)$; i.e.

$$
m_{2}(v) f(v)=\sum_{w \sim v} f(w) .
$$

Then, we have

$$
\begin{aligned}
\sum_{y \sim x} f(\varphi(y)) & =\sum_{y \sim x, \varphi(y)=v} f(\varphi(y))+\sum_{y \sim x, \varphi(y)=w, w \sim v} f(\varphi(y)) \\
& =m_{v}(x) f(v)+\sum_{w \sim v} \#\left\{y \in V_{1} ; y \sim x, \varphi(y)=w\right\} f(w) \\
& =m_{v}(x) f(v)+k(x) \sum_{w \sim v} f(w) \\
& =\left(m_{v}(x)+k(x) m_{2}(v)\right) f(v) \\
& =m_{1}(x) f(v) \\
& =m_{1}(x) f \circ \varphi(x),
\end{aligned}
$$

which implies that $f \circ \varphi$ is harmonic at $x$.

On combining Lemmas 2.6, 2.7 and 2.11, we obtain Theorem 2.5.

We give here applications of Theorem 2.5.

Let $\varphi: \quad V_{1} \rightarrow V_{2}$ be a harmonic morphism of $G_{1}$ to $G_{2}$. By Theorem 2.5, it is equivalent that $\varphi$ is horizontally conformal. By the condition (a) of horizontal conformality, for each edge $e \in E_{1}$ of $G_{1}$, the map $\varphi$ sends it to an edge in $E_{2}$ or one vertex of $V_{2}$. It is easy to see that the condition (b) is equivalent to the following condition.

(b') For each $x \in V_{1}, y=\varphi(x) \in V_{2}$ and each $e^{\prime} \in E_{2}$ incident to $y$, $\#\left\{e \in E_{1} ; \varphi(e)=e^{\prime}\right.$, e is incident to $\left.x\right\}$ depends only on $x$, but not on $e^{\prime} \in E_{2}$ incident to $x$. Note that this number is equal to $k(x), x \in V_{1}$.

Lemma 2.12. Let $\varphi: V_{1} \rightarrow V_{2}$ be a harmonic morphism of $G_{1}$ to $G_{2}$. Then for each $e^{\prime} \in E_{2}, \#\left\{e \in E_{1} ; \varphi(e)=e^{\prime}\right\}$ is independent of $e^{\prime}$. 
Proof. Assume that two edges $e^{\prime}$ and $e^{\prime \prime}$ in $E_{2}$ are incident to a common vertex $y \in V_{2}$. Due to the condition $\left(\mathrm{b}^{\prime}\right)$, for each $x \in \varphi^{-1}(y)$ we have

$$
\#\left\{e \in E_{1} ; \varphi(e)=e^{\prime} \text {, incident to } x\right\}=\#\left\{\tilde{e} \in E_{1} ; \varphi(\tilde{e})=e^{\prime \prime} \text {, incident to } x\right\} \text {. }
$$

Therefore,

$$
\begin{aligned}
\#\left\{e \in E_{1} ; \varphi(e)=e^{\prime}\right\} & =\sum_{x \in \varphi^{-1}(y)} \#\left\{e \in E_{1} ; \varphi(e)=e^{\prime}, \text { incident to } x\right\} \\
& =\sum_{x \in \varphi^{-1}(y)} \#\left\{\tilde{e} \in E_{1} ; \varphi(\tilde{e})=e^{\prime \prime}, \text { incident to } x\right\} \\
& =\#\left\{\tilde{e} \in E_{1} ; \varphi(\tilde{e})=e^{\prime \prime}\right\} .
\end{aligned}
$$

We assume that $G$ is connected and so, given two edges $e^{\prime}$ and $e^{\prime \prime}$ in $E_{2}$, there exists edges $e_{i}^{\prime}$ in $E_{2}(i=1,2, \cdots, n+1)$ with $e_{i}^{\prime}=e^{\prime}$ and $e_{n+1}^{\prime}=e^{\prime \prime}$ such that each two $e_{i}^{\prime}$ and $e_{i+1}^{\prime}$ are contiguous by a common edge in $V_{2}$. Applying the above argument to $e_{i}^{\prime}$ and $e_{i+1}^{\prime}$ in succession, we obtain Lemma 2.12 .

We have the following result.

Theorem 2.13. Let $\varphi: V_{1} \rightarrow V_{2}$ be a harmonic morphism of $G_{1}$ to $G_{2}$. Then the pull-back by $\varphi$ induces an injection $\varphi *: \mathbf{H}^{1}\left(G_{2}\right) \rightarrow \mathbf{H}^{1}\left(G_{1}\right)$, where $\mathbf{H}^{1}\left(G_{i}\right)$ is the space of harmonic 1-forms of graphs $G_{i}=\left(V_{i}, E_{i}\right)$.

Proof. We first have to check that the pull-back can induce the homomorphism of $\mathbf{H}^{1}\left(G_{2}\right)$ into $\mathbf{H}^{1}\left(G_{1}\right)$. Due to the condition $\left(\mathrm{b}^{\prime}\right), \#\left\{e \in \mathbf{E}_{1} ; \varphi(e)=e^{\prime}\right\}$ depends only on $x$, but not in $e^{\prime} \in \mathbf{E}_{2}$ (cf. Lemma 2.12). We denote by $k_{x}$ this number. Then for $\omega \in A^{1}\left(G_{2}\right)$, i.e. a function on $\mathbf{E}_{2}$ satisfying $\omega(e)=-\omega(\bar{e})$, we have

$$
\begin{aligned}
\sum_{e \in \mathbf{E}_{1}, o(e)=x} \omega(\varphi(e)) & =\sum_{e^{\prime} \in \mathbf{E}_{2}, o\left(e^{\prime}\right)=y} \sum_{e \in \mathbf{E}_{1}, \varphi(e)=e^{\prime}} \omega(\varphi(e)) \\
& =k_{x}\left(\sum_{e^{\prime} \in \mathbf{E}_{2}, o\left(e^{\prime}\right)=y} \omega\left(e^{\prime}\right)\right)
\end{aligned}
$$

Therefore, if $\omega$ satisfies $\delta \omega=0$, for $x \in V_{1}$ and $y=\varphi(x) \in V_{2}$,

$$
\begin{aligned}
m(x) \delta\left(\varphi^{*} \omega\right)(x) & =-\sum_{e \in \mathbf{E}_{1}, o(e)=x} \varphi^{*} \omega(e) \\
& =-\sum_{e \in \mathbf{E}_{1}, o(e)=x} \omega(\varphi(e)) \\
& =-k_{x}\left(\sum_{e^{\prime} \in \mathbf{E}_{2}, o\left(e^{\prime}\right)=y} \omega\left(e^{\prime}\right)\right) \\
& =k_{x} m(y) \delta \omega(y)=0,
\end{aligned}
$$


which implies that $\varphi$ induces a homomorphism $\varphi^{*}: \mathbf{H}^{1}\left(G_{2}\right) \rightarrow \mathbf{H}^{1}\left(G_{1}\right)$. To prove that it is an injection, we assume $\varphi^{*} \omega(e)=0$, for all $e \in \mathbf{E}_{1}$, and so we have $\omega(\varphi(e))=0$ for all $e \in \mathbf{E}_{1}$. But since $\varphi: \mathbf{E}_{1} \rightarrow \mathbf{E}_{2}$ is a surjection, we have $\omega\left(e^{\prime}\right)=0$ for all $e^{\prime} \in \mathbf{E}_{2}$; that is, $\omega=0$.

COROLlary 2.14. Let $\varphi: V_{1} \rightarrow V_{2}$ be a harmonic morphism. Then the first Betti numbers and Euler numbers satisfy $b_{1}\left(G_{1}\right) \geq b_{1}\left(G_{2}\right)$ and $\chi\left(G_{2}\right) \geq \chi\left(G_{1}\right)$.

Proof. It is clear due to Theorem 2.13; indeed, we have

$$
b_{1}\left(G_{1}\right)=\operatorname{dim} \mathbf{H}^{1}\left(G_{1}\right) \geq \operatorname{dim} \mathbf{H}^{1}\left(G_{2}\right)=b_{1}\left(G_{2}\right),
$$

and $\chi\left(G_{2}\right) \geq \chi\left(G_{1}\right)$, since $b_{1}\left(G_{i}\right)=1-\chi\left(G_{i}\right)$.

3. The Laplacians and Green Kernels of Harmonic Morphisms. We first define the vertical and horizontal Laplacians for harmonic morphisms and apply them to estimate Green kernels.

Definition 3.1. Let $\varphi: V_{1} \rightarrow V_{2}$ be a harmonic morphism of $G_{1}$ to $G_{2}$.

(1) The vertical Laplacian is defined by

$$
\Delta_{v} f(x)=f(x)-\frac{1}{m_{v}(x)} \sum_{\substack{z \sim x \\ z \in \varphi^{-1}(\varphi(x))}} f(z),
$$

where $m_{v}(x)$ is the vertical degree of $x$ (cf. Definition 2.4).

(2) The horizontal Laplacian is defined also by

$$
\Delta_{h} f(x)=f(x)-\frac{1}{m_{h}(x)} \sum_{\substack{z \sim 1 \\ z \notin \varphi^{-1}(\varphi(x))}} f(z),
$$

where $m_{h}(x)$ is the horizontal degree of $x$ (cf. Definition 2.4).

Proposition 3.2. (1) $\Delta=\frac{m_{v}}{m} \Delta_{v}+\frac{m_{h}}{m} \Delta_{h}$ holds.

(2) Moreover, for $f \in C\left(V_{2}\right)$, we have

$$
\Delta_{v}(f \circ \varphi)=0, \quad \Delta_{h}(f \circ \varphi)=(\Delta f) \circ \varphi, \quad \Delta(f \circ \varphi)=\frac{m_{h}}{m}(\Delta f) \circ \varphi .
$$

Proof. Since $m(x)=m_{v}(x)+m_{h}(x)$, for each $x \in V_{1}$, and

$$
\left\{z \in V_{1} ; z \sim x\right\}=\left\{z \in \varphi^{-1}(\varphi(x)) ; z \sim x\right\} \bigcup\left\{z \notin \varphi^{-1}(\varphi(x)) ; z \sim x\right\},
$$

we have (1) immediately. For (2), note that for $z \in \varphi^{-1}(\varphi(x))$ with $z \sim x, f \circ \varphi(z)=$ $f \circ \varphi(x)$, and by the definition of $m_{v}(x)$, we have $\Delta_{v}(f \circ \varphi)(x)=0$, for all $x \in V_{1}$. For $\Delta_{h}(f \circ \varphi)$, we have 


$$
\begin{aligned}
\Delta_{h}(f \circ \varphi)(x) & =f \circ \varphi(x)-\frac{1}{m_{h}(x)} \sum_{\substack{z \sim x \\
z \notin \varphi^{-1}(\varphi(x))}} f \circ \varphi(z) \\
& =f \circ \varphi(x)-\frac{1}{m_{h}(x)} \sum_{y^{\prime} \sim \varphi(x)} \sum_{\substack{z \sim x \\
z \in \varphi^{-1}\left(y^{\prime}\right)}} f \circ \varphi(z) .
\end{aligned}
$$

But here, since $f \circ \varphi(z)=f \circ \varphi\left(y^{\prime}\right)$ for $z \in \varphi^{-1}\left(y^{\prime}\right)$, we have

$$
\begin{aligned}
\sum_{\substack{z \sim x \\
z \in \varphi^{-1}\left(y^{\prime}\right)}} f \circ \varphi(z) & =\#\left\{z \in \varphi^{-1}\left(y^{\prime}\right) ; z \sim x\right\} f \circ \varphi\left(y^{\prime}\right) \\
& =\frac{m_{h}(x)}{m(\varphi(x))} f \circ \varphi\left(y^{\prime}\right) .
\end{aligned}
$$

Hence, we have $\Delta_{h}(f \circ \varphi)(x)=(\Delta f)(\varphi(x))$. It is clear that $\Delta_{v}(f \circ \varphi)=0$, and so

$$
\Delta(f \circ \varphi)(x)=\frac{m_{h}(x)}{m(x)} \Delta_{h}(f \circ \varphi)(x)=\frac{m_{h}(x)}{m(x)}(\Delta f)(\varphi(x)) .
$$

Proposition 3.2 is proved.

Now let us recall the heat kernel and Green kernel for an infinite graph $G=(V, E)$. Let $p(x, y), x, y \in V$, be the one-step transition probability of a random walk on $G$ from $x$ to $y$, that is,

$$
p(x, y)= \begin{cases}\frac{1}{m(x)} & y \sim x \\ 0 & \text { otherwise }\end{cases}
$$

Let us define inductively

$$
p^{n+1}(x, y)=\sum_{z \in V} p(x, z) p^{n}(z, y)
$$

where $p^{0}(x, y)$ is defined by

$$
p^{0}(x, y)= \begin{cases}1 & x=y \\ 0 & x \neq y\end{cases}
$$

We call $p^{n}(x, y)$ the heat kernel and the Green kernel $G(x, y)$ is defined by

$$
G(x, y)=\sum_{n=0}^{\infty} p^{n}(x, y),
$$

for $x, y \in V$. The graph is said to be transient if $G(x, y)<\infty$, for all $x, y \in V$, and recurrent otherwise. Note that if the graph is transient, the Green kernel satisfies 


$$
\Delta_{x} G(x, y)=p^{0}(x, y)
$$

Our next main result is as follows.

Theorem 3.3. Let $G_{i}=\left(V_{i}, E_{i}\right), i=1,2$, be two infinite graphs, and $G_{i}$, their Green kernels. Let $\varphi: V_{1} \rightarrow V_{2}$ be a harmonic morphism of $G_{1}$ to $G_{2}$. Then we have

$$
G_{1}(x, y) \leq \frac{m(y)}{m_{h}(y)} G_{2}(\varphi(x), \varphi(y))
$$

for all $x$ and $y \in V_{1}$.

To prove this theorem, we need the maximum principle.

Lemma 3.4. Let $D$ be a finite connected subset in a graph $G=(V, E)$ and $f, a$ function on $D \cup \partial D$ satisfying

$$
\Delta f \geq 0 \text { on } D, \text { and } f \geq 0 \text { on } \partial D .
$$

Then $f \geq 0$ on $D \cup \partial D$.

For a proof, see [2] or [9, Lemma 1.3].

Lemma 3.5. Let $G=(V, E)$ be an infinite graph and $G(x, y)$ be its Green kernel. For a connected finite subset $D$ with boundary $\partial D$ in $V$, let us denote by $G_{D}(x, y)$ its Green kernel; (see [9, Definition 4.3]). Fix a vertex $p \in V$ and let $B_{r}(p)=$ $\{x \in V ; d(x, p)<r\}$, for all $r>0$. Then we have

$$
\lim _{r \rightarrow \infty} G_{\mathrm{B}_{r}(p)}(x, y)=G(x, y)
$$

for every $x$ and $y \in V$.

For a proof, see [9, Theorem 4.6].

Proof of Theorem 3.3. For $p \in V_{1}$ and $r>0$, let us define

$$
B_{r}^{1}(p)=\left\{x \in V_{1} ; d(x, p)<r\right\}, \quad B_{r}^{2}(\varphi(p))=\left\{y \in V_{2} ; d(y, \varphi(p))<r\right\} .
$$

By Lemma 2.6, we have

$$
\varphi\left(B_{r}^{1}(p)\right) \subset B_{r}^{2}(\varphi(p))
$$

Denote by $G_{1, r}(x, y)=G_{1 B_{r}^{1}(p)}(x, y), x, y \in V_{1}$, and $G_{2, r}(z, w)=G_{2 B_{r}^{2}(\varphi(p))}(z, w), z, w \in$ $V_{2}$, the Green kernel for $B_{r}^{1}(p)$ and $B_{r}^{2}(\varphi(p))$, respectively.

Now let us consider for a fixed $y \in B_{r}^{1}(p)$ the function $u$ on $B_{r}^{1}(p)$, defined by

$$
u(x)=-G_{1, r}(x, y)+\frac{m(y)}{m_{h}(y)} G_{2, r}(\varphi(x), \varphi(y)) .
$$

Then we have the following result. 
Lemma 3.6. (1) The function $u$ on $B_{r}^{1}(p)$ satisfies

$$
u(x) \geq 0, \quad \text { for all } x \in \partial B_{r}^{1}(p) .
$$

(2) Moreover, we have

$$
\Delta u(x) \geq 0, \quad \text { for all } x \in B_{r}^{1}(p) .
$$

Proof. For (1), we have $G_{1, r}(x, y)=0$ for all $x \in \partial B_{r}^{1}(p)$ by definition (cf. [9, Proposition 4.4]). Therefore, $u(x) \geq 0$ because of positivity of the Green kernels. For (2), let $x \in \partial B_{r}^{1}(p)$. Making use of Proposition 3.2, we obtain

$$
\begin{aligned}
\Delta_{1} u(x) & =-\Delta_{1, x} G_{1, r}(x, y)+\frac{m(y)}{m_{h}(y)} \Delta_{1, x} G_{2, r}(\varphi(x), \varphi(y)), \\
& =-\Delta_{1, x} G_{1, r}(x, y)+\frac{m(y)}{m_{h}(y)} \frac{m_{h}(x)}{m(x)}\left(\Delta_{2} G_{2, r}\right)(\varphi(x), \varphi(y)) .
\end{aligned}
$$

Therefore, if $x \neq y$, we have,

$$
\Delta_{1} u(x)= \begin{cases}0+0=0, & \text { (if } \varphi(x) \neq \varphi(y)), \\ 0+\frac{m(y)}{m_{h}(y)} \frac{m_{h}(x)}{m(x)}>0, & (\text { if } \varphi(x)=\varphi(y)) .\end{cases}
$$

If $x=y$, we have

$$
\Delta_{1} u(x)=-1+\frac{m(x)}{m_{h}(x)} \frac{m_{h}(x)}{m(x)}=0 .
$$

Therefore, we obtain $\Delta_{1} u(x) \geq 0$ for $x \in B_{r}^{1}(p)$.

Proof of Theorem 3.3 (continued). By the maximum principle (Lemma 3.4), we obtain

$$
u \geq 0, \quad \text { on } \quad B_{r}^{1}(p) \cup \partial B_{r}^{1}(p)
$$

Therefore, we obtain that, for $x, y \in B_{r}^{1}(p) \cup \partial B_{r}^{1}(p)$,

$$
G_{1, r}(x, y) \leq \frac{m(y)}{m_{h}(y)} G_{2, r}(\varphi(x), \varphi(y))
$$

Letting $r \rightarrow \infty$, we obtain that for $x, y \in V_{1}$,

$$
G_{1}(x, y) \leq \frac{m(y)}{m_{h}(y)} G_{2}(\varphi(x), \varphi(y))
$$

We have proved Theorem 3.3.

REMARK 3.7. It is not true, in general, that the heat kernels $p_{i}^{n}(x, y)$ for the harmonic morphism $\varphi: V_{1} \rightarrow V_{2}$ of $G_{1}$ to $G_{2}$ satisfy 


$$
p_{1}^{n}(x, y) \leq \frac{m(y)}{m_{h}(y)} p_{2}^{n}(\varphi(x), \varphi(y))
$$

for each $n=1,2 \cdots$ and $x, y \in V_{1}$.

Indeed, if we consider the example given by Figure 2, we have $p_{1}^{1}\left(x, y_{0}\right)=\frac{1}{4}$ and
Ind $n$. $p_{2}^{1}\left(\varphi(x), \varphi\left(y_{0}\right)\right)=0$. However, Theorem 3.3 shows that

$$
G_{1}\left(x, y_{0}\right) \leq \frac{4}{3} G_{2}\left(\varphi(x), \varphi\left(y_{0}\right)\right), \quad G_{1}(x, y) \leq G_{2}(\varphi(x), \varphi(y)), \quad\left(y \neq y_{0}\right) .
$$

4. Examples and applications. In this section, we give examples of harmonic morphisms and show applications in estimates of the Green kernels for infinite trees.

4.1. Coverings. A mapping $\varphi: V_{1} \rightarrow V_{2}$ is a covering of $G_{1}\left(V_{1}, E_{1}\right)$ to $G_{2}=\left(V_{2}, E_{2}\right)$ if $\varphi: V_{1} \rightarrow V_{2}$ is surjective, $m(x)=m(\varphi(x))$ for all $x \in V_{1}$ and for each $x$ and $z \in V_{1}, \sim x$ is equivalent to $\varphi(z) \sim \varphi(x)$. A covering is locally a distance preserving mapping of $V_{1}$ to $V_{2}$. By definition, a covering is a harmonic morphism.

4.2 NEPS. Let us recall the definition of non-complete extended p-sum, we say briefly, NEPS (cf. [7]): Let $G_{1}, \cdots, G_{n}$ be $n$ graphs. Take a non-empty subset $B$ in the $n$-product space $\{0,1\} \times \cdots \times\{0,1\}$ satisfying that $B$ does not include $(0, \cdots, 0)$. Let $H$ be a graph whose set of vertices, $V(H)$ is the product space $V(H)=V\left(G_{1}\right) \times \cdots \times V\left(G_{n}\right) . H$ is said to be NEPS with basis $B$ if the adjacency relation is defined as follows. For $\left(u_{1}, \cdots, u_{n}\right)$ and $\left(v_{1}, \cdots, v_{n}\right) \in V(H),\left(u_{1}, \cdots, u_{n}\right) \sim$ $\left(v_{1}, \cdots, v_{n}\right)$ if and only if there exists an element $b=\left(b_{1}, \cdots, b_{n}\right) \in B$ such that

$$
\begin{cases}u_{i}=v_{i} & \text { if } b_{i}=0, \\ u_{i} \sim v_{i} \text { in } G_{i} & \text { if } b_{i}=1\end{cases}
$$

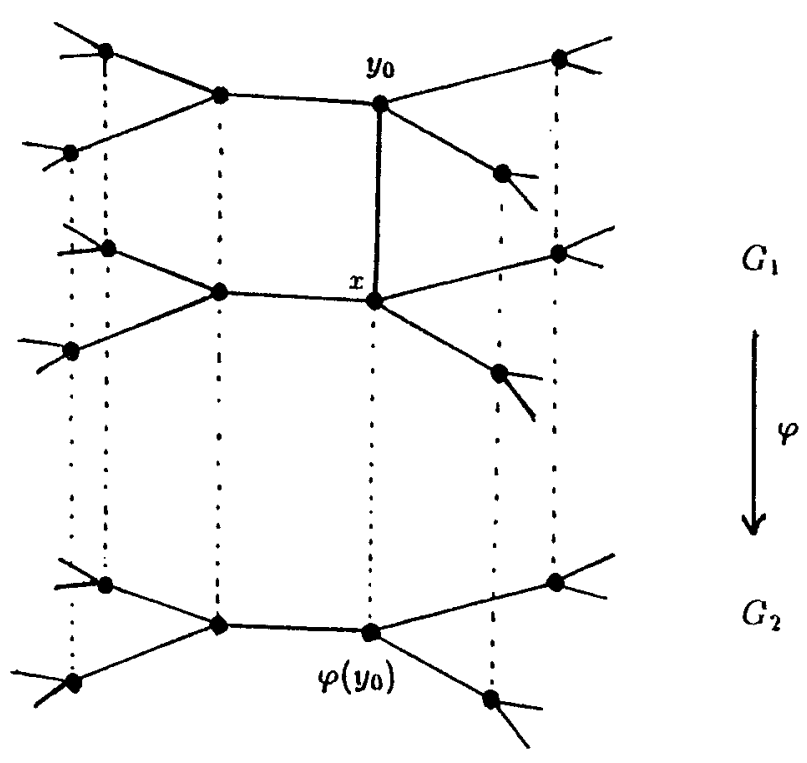

Figure 2 
Then we obtain the following result.

Proposition 4.1. Let $H$ be NEPS of $G_{1}, \cdots, G_{n}$ with basis B. For each $i=1, \cdots, n$, the $i$-th projection of $H$ onto $G_{i}$, defined by $\varphi_{i}\left(u_{i}, \cdots, u_{n}\right)=u_{i}$, is a harmonic morphism.

Proof. We only have to see horizontal conformality of $\varphi_{i}$. It is clear that the condition (a) is satisfied. To prove (b) observe that for each $u_{i} \in V\left(G_{i}\right)$,

$$
\varphi^{-1}\left(u_{i}\right)=\left\{\left(w_{1}, \cdots, w_{i-1}, u_{i}, w_{i+1}, \cdots, w_{n}\right) ; w_{j} \in V\left(G_{j}\right), j \neq i\right\} .
$$

Let us take an element $x=\left(z_{1}^{0}, \cdots, z_{i-1}^{0}, u_{i}, z_{i+1}^{0}, \cdots, z_{n}^{0}\right) \in \varphi^{-1}\left(u_{i}\right)$ and take $v_{i} \in G_{i}$ with $v_{i} \sim u_{i}$. The set $\left\{z \in \varphi^{-1}\left(v_{i}\right) ; z \sim x\right\}$ coincides with

$$
\begin{gathered}
\left\{z=\left(w_{1}, \cdots, w_{i-1}, v_{i}, w_{i+1}, \cdots w_{n}\right) ; z \sim x\right\} \\
=\left\{\left(w_{1}, \cdots, w_{i-1}, v_{i}, w_{i+1}, \cdots, w_{n}\right) ; \exists\left(b_{1}, \cdots, b_{n}\right) \in B, b_{i}=1,\right. \\
\left.w_{j}=z_{j}^{0}\left(b_{j}=0, j \neq i\right), w_{j} \sim z_{j}^{0}\left(b_{j}=1, j \neq i\right)\right\} .
\end{gathered}
$$

Let $B^{\prime}$ be a subset of the $(n-1)$-product $\{0,1\} \times \cdots \times\{0,1\}$ defined by

$$
B^{\prime}=\left\{\left(b_{1}, \cdots, b_{i-1}, b_{i+1}, \cdots, b_{n}\right) ;\left(b_{1}, \cdots, b_{i-1}, 1, b_{i+1}, \cdots, b_{n}\right) \in B\right\}
$$

Then $\#\left\{z \in \varphi^{-1}\left(v_{i}\right) ; z \sim x\right\}$ coincides with

$$
\#\left\{\left(w_{1}, \cdots, w_{i-1}, w_{i+1}, \cdots, w_{n}\right) ; \exists b^{\prime} \in B^{\prime}, w_{j}=z_{j}^{0}\left(b_{j}^{\prime}=0\right), w_{j} \sim z_{j}^{0}\left(b_{j}^{\prime}=1\right)\right\},
$$

which does not depend on $v_{i}$. We have (b).

4.3. Collapsing. We give another type of harmonic morphism, which has no analogy in Riemannian geometry. Let $G=(V, E)$ be a graph, and $V^{\prime}$ be a subset of $V$ satisfying the condition that $V^{\prime}$ is connected to $V-V^{\prime}$ through only one vertex $x^{\prime} \in V^{\prime}$. Let $\left\{y_{1}, \cdots, y_{n}\right\}$ be the set of vertices in $V-V^{\prime}$ being connected to $x^{\prime}$. The collapsing is to produce a new graph $G_{2}=\left(V_{2}, E_{2}\right)$ by shrinking $V^{\prime}$ into one point, say, $p$, and connecting all $y_{i}$ to $p$. Let us define the collapsing of $G$ to $G_{2}, \varphi: V \rightarrow V_{2}$ by

$$
\varphi(x)= \begin{cases}x & x \in V-V^{\prime} \\ p & x \in V^{\prime}\end{cases}
$$

Then we have the following result.

Proposition 4.2. The collapsing $\varphi: V \rightarrow V_{2}$ of $G$ to $G_{2}$ is a harmonic morphism.

Proof. We only have to prove for $\varphi$ the condition (b) of the horizontal conformality. First, let $y \in V_{2}$ with $y \neq y_{r}, y \neq p$. Then $\varphi^{-1}(y)=\{y\}$. Since 
$\left\{y^{\prime} ; y^{\prime} \sim y\right\}$ does not intersect $V^{\prime}$, we have $\left\{z \in \varphi^{-1}\left(y^{\prime}\right) ; z \sim x\right\}=\left\{y^{\prime}\right\}$. Thus $\#\left\{z \in \varphi^{-1}\left(y^{\prime}\right) ; z \sim x\right\}=1$, which is independent on $y^{\prime} \in V_{2}$ with $y^{\prime} \sim y$.

Second, let $y=y_{i}$ for some $i=1, \cdots, n$. Then $\varphi^{-1}(y)=\left\{y_{i}\right]$. Let $y^{\prime} \in V_{2}$ with $y^{\prime} \sim y$. Then $y^{\prime} \in V-V^{\prime}$ or $y^{\prime}=p$. In the case $y^{\prime} \in V-V^{\prime}$, we have $\left\{z \in \varphi^{-1}\left(y^{\prime}\right) ; z \sim x\right\}=\left\{y^{\prime}\right\}$, which implies that $\#\left\{z \in \varphi^{-1}\left(y^{\prime}\right) ; z \sim x\right\}=1$. In the case $y^{\prime}=p$, we have $\left\{z \in \varphi^{-1}\left(y^{\prime}\right) ; z \sim x\right\}=\left\{z \in \varphi^{-1}(p) ; z \sim y_{i}\right\}=\left\{x^{\prime}\right\}$. We have $\#\left\{z \in \varphi^{-1}\left(y^{\prime}\right) ; z \sim x\right\}=1$, which does not depend on a choice of $y^{\prime} \in V_{2}$ with $y^{\prime} \sim y$ in this case.

Third, let $y=p$. Then $\varphi^{-1}(y)=\{p\} \cup\left\{x \in V^{\prime} ; x \neq p\right\}$. In the case $x \in V^{\prime}$ with $x \neq p,\left\{y^{\prime} \in V_{2} ; y^{\prime} \sim p\right\}=\left\{y_{i}\right\}$. Then, $\left\{z \in \varphi^{-1}\left(y^{\prime}\right) ; z \sim x\right\}=\left\{y_{i} ; y_{i} \sim x\right\}$ is an empty set, so that $\#\left\{z \in \varphi^{-1}\left(y^{\prime}\right) ; z \sim x\right\}=0$ is independent of the choice of $y^{\prime}$. In the case $x=p,\left\{y^{\prime} \in V_{2} ; y^{\prime} \sim p\right\}=\left\{y_{i} ; i=1, \cdots, n\right\}$, so that for $y^{\prime}=y_{i},\left\{z \in \varphi^{-1}\left(y^{\prime}\right) ; z \sim x\right\}=$ $\left\{y_{i}, y_{i} \sim p\right\}=\left\{y_{i}\right\}$. Hence $\#\left\{z \in \varphi^{-1}\left(y^{\prime}\right) ; z \sim x\right\}=1$, which in independent of the choice of $y^{\prime}$ with $y^{\prime} \sim p$. We have established (b).

Applying Theorem 3.3, we obtain the next result.

Theorem 4.3. Let $G=(V, E)$ be an infinite tree. Assume that $W$ is a subset of $V$ satisfying

(a) $V-W$ is connected and an infinite set, and

(b) $m(x) \geq k>3$, for all $x \in V-W$.

Then we have

$$
G(x, y) \leq \frac{k-2}{k-3}\left(\frac{1}{k-2}\right)^{d_{2}(\varphi(x), \varphi(y))}(x, y \in V)
$$

where we take $V^{\prime}=W \cup\left\{x^{\prime}\right\}, x^{\prime}$ is a unique vertex in $V-W$ connected to some vertex in $W$, and $\varphi: V \rightarrow V_{2}$ is a collapsing given by

$$
\varphi(x)= \begin{cases}x & x \in V-V^{\prime} \\ p & x \in V^{\prime}\end{cases}
$$

where $p \in V^{\prime}$ is a unique vertex connecting to $V-V^{\prime}$, and $d_{2}$ is the distance of the graph $G_{2}=\left(V_{2}, E_{2}\right)$.

Proof. Let $G_{2}$ be the resulting collapsing, which is a tree satisfying

$$
m(x) \geq k-1, \quad \text { for all } x \in V_{2} .
$$

Due to the above Theorem 3.3 and Theorem 7.2 in [9], we obtain

$$
G(x, y) \leq G_{2}(\varphi(x), \varphi(y)) \leq G_{\mathrm{T}_{k-1}}(\varphi(x), \varphi(y))=\frac{k-2}{k-3}\left(\frac{1}{k-2}\right)^{d_{2}(\varphi(x), \varphi(y))} .
$$

We have established Theorem 4.3.

The following Figure 3 illustrates the situation in Theorem 4.3.

REMARK 4.4. (a) In general, a harmonic morphism is not rough isometric in the sense of Kanai [5], [10]. In fact, $\varphi: V_{1} \rightarrow V_{2}$ is rough isometric of $G_{1}$ to $G_{2}$ if and only if there exist positive constants $A$ and $B$ satisfying 


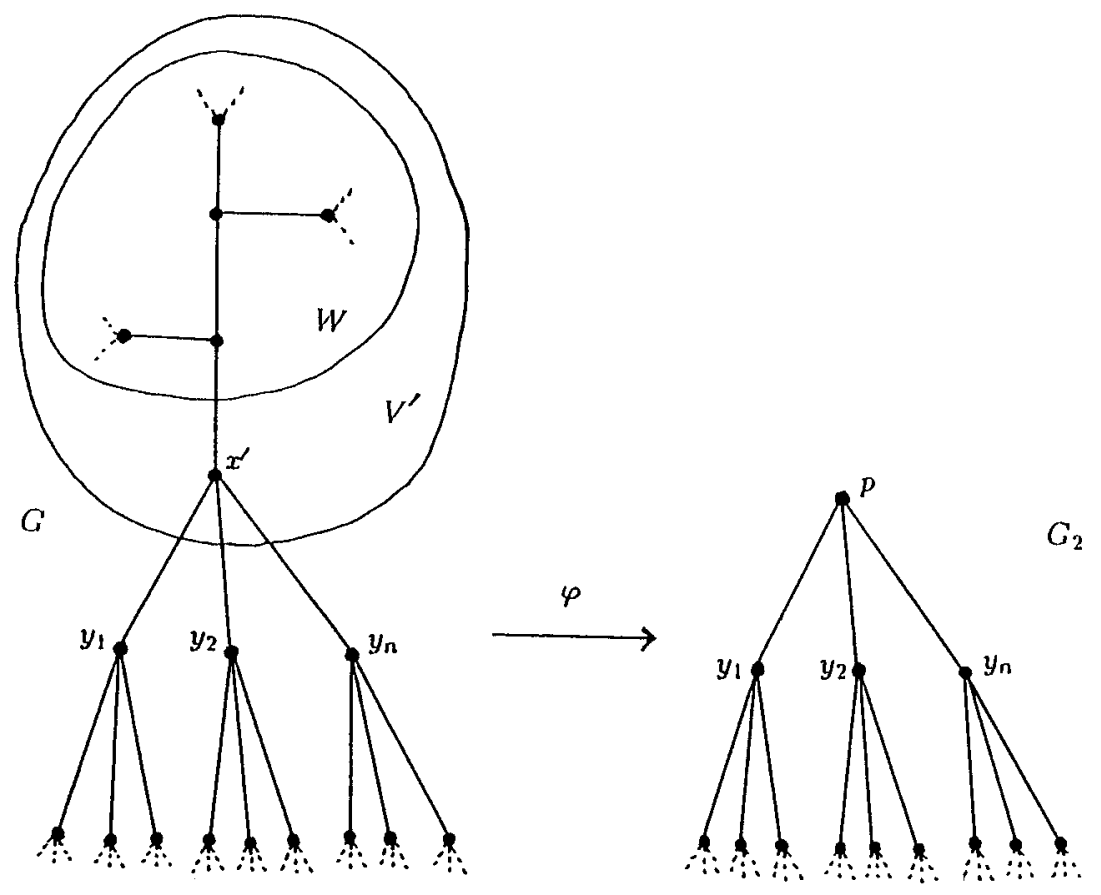

Figure 3

$$
A^{-1} d_{1}(x, y)-B \leq d_{2}(\varphi(x), \varphi(y)) \leq A d_{1}(x, y)+B, d_{2}\left(x^{\prime}, \varphi\left(V_{1}\right)\right) \leq B
$$

for all $x, y \in V_{1}$ and $x^{\prime} \in V_{2}$, where $d_{i}$ is the distance of $G_{i}, i=1,2$. But in our harmonic morphism, one can change the vertical part, i.e. making the collapsing part $V^{\prime}$ large, so that $d_{1}(x, y)$ with $x, y \in V^{\prime}$ tends to infinity, but keeping $d_{2}(\varphi(x), \varphi(y))=0$.

(b) We have not considered the case $k=3$ in Theorem 4.3 .

Acknowledgements. The author would like to express his gratitude to Professor Anand who gave many useful comments, Mr. Tsuruta who corrected mistakes in the first draft, and the referee who improved the proof of Theorem 2.5.

\section{REFERENCES}

1. G. Besson, A Kato type inequality for Riemannian submersions with tottally geodesic fibers, Ann. Global Anal. Geom. 4 (1986), 273-289.

2. J. Dodziuk, Difference equations, isoperimetric inequality and transience of certain random walks, Trans. Amer. Math. Soc. 284 (1984), 787-794.

3. B. Fugledge, Harmonic morphisms between Riemannian manifolds, Ann. Inst. Fourier (Grenoble) 28 (1978), 107-144.

4. T. Ishihara, A mapping of Riemannian manifolds which preserves harmonic functions, J. Math. Kyoto Univ. 19 (1979), 215-229.

5. M. Kanai, Rough isometries and the parabolicity of Riemannian manifolds, J. Math. Soc. Japan 38 (1986), 227-238.

6. A. Kasue and T. Washio, Growth of equivariant harmonic maps and harmonic morphisms, Osaka J. Math. 27 (1990), 899-928. 
7. B. Mohar and W. Woess, A survey on spectra of infinite graphs, Bull. London Math. Soc. 21 (1989), 209-234.

8. H. Urakawa, Spectral geometry of the second variation operator of harmonic maps, Illinois J. Math. 33 (1989), 250-267.

9. H. Urakawa, Heat kernel and Green kernel comparison theorems for infinite graphs, J. Functional Analysis 146 (1997), 206-235.

10. H. Urakawa, Eigenvalue comparison theorems of the discrete Laplacian for a graph, Goem. Dedicata 74 (1999), 95-112.

11. W. Woess, Random walks on infinite graphs and groups. A survey on selected topics, Bull. London Math. Soc. 26 (1994), 1-60. 